Malmberg, Isolde; Nestler, Emanuel; Retzlaff-Fürst, Carolin Qualitäten der Mentor*innenqualifizierung M-V. Eine Design Based Research Studie zu einem Lernbegleitungsprogramm an der Schnittstelle zwischen \title{
Schule und Hochschule
}

Hesse, Florian [Hrsg.]; Lütgert, Will [Hrsg.]: Auf die Lernbegleitung kommt es an! Konzepte und Befunde zu Praxisphasen in der Lehrerbildung. Bad Heilbrunn : Verlag Julius Klinkhardt 2020, S. 81-106

Quellenangabe/ Reference:

Malmberg, Isolde; Nestler, Emanuel; Retzlaff-Fürst, Carolin: Qualitäten der

Mentor*innenqualifizierung M-V. Eine Design Based Research Studie zu einem

Lernbegleitungsprogramm an der Schnittstelle zwischen Schule und Hochschule - In: Hesse, Florian [Hrsg.]; Lütgert, Will [Hrsg.]: Auf die Lernbegleitung kommt es an! Konzepte und Befunde zu Praxisphasen in der Lehrerbildung. Bad Heilbrunn : Verlag Julius Klinkhardt 2020, S. 81-106 - URN: urn:nbn:de:0111-pedocs-200805 - DOI: 10.25656/01:20080

https://nbn-resolving.org/urn:nbn:de:0111-pedocs-200805

https://doi.org/10.25656/01:20080

in Kooperation mit / in cooperation with:

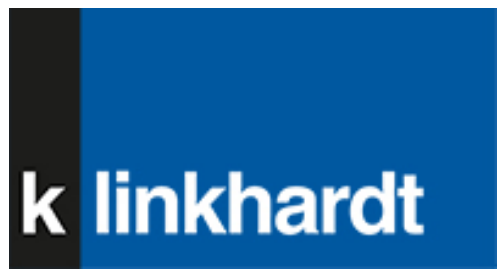

http://www.klinkhardt.de

\section{Nutzungsbedingungen}

Dieses Dokument steht unter folgender Creative Commons-Lizenz: http://creativecommons.org/licenses/by-nc-sa/4.0/deed.de - Sie dürfen das Werk bzw. den Inhalt unter folgenden Bedingungen vervielfältigen, verbreiten und öffentlich zugänglich machen sowie Abwandlungen und Bearbeitungen des Werkes bzw. Inhaltes anfertigen: Sie müssen den Namen des Autors/Rechteinhabers in der von ihm festgelegten Weise nennen. Dieses Werk bzw, der Inhalt darf nicht für kommerzielle Zwecke verwendet werden. Die neu entstandenen Werke bzw. Inhalte dürfen nur unter Verwendung von Lizenzbedingungen weitergegeben werden, die mit denen dieses Lizenzvertrages identisch oder vergleichbar sind.

Mit der Verwendung dieses Dokuments erkennen Sie die Nutzungsbedingungen an

\section{Terms of use}

This document is published under following Creative Commons-License: http://creativecommons.org/licenses/by-nc-sa/4.0/deed.en - You may copy, distribute and transmit, adapt or exhibit the work in the public and alter, transform or change this work as long as you attribute the work in the manner specified by the author or licensor. You are not allowed to make commercial use of the work. If you alter, transform, or change this work in any way, you may distribute the resulting work only under this or a comparable license.

By using this particular document, you accept the above-stated conditions of use.

\section{Kontakt / Contact:}

peDOcs

DIPF | Leibniz-Institut für Bildungsforschung und Bildungsinformation

Informationszentrum (IZ) Bildung

E-Mail: pedocs@dipf.de

Internet: www.pedocs.de

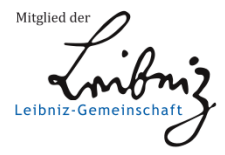


Isolde Malmberg, Emanuel Nestler und Carolin Retzlaff-Fürst

\section{Qualitäten der Mentor*innenqualifizierung M-V. Eine Design Based Research Studie zu einem Lernbegleitungsprogramm an der Schnittstelle zwischen Schule und Hochschule}

Mentorinnen und Mentoren sind Lernbegleitende an einer wichtigen Stelle: Sie begleiten am sensiblen Übergang, an der Schnittstelle zwischen Hochschule und Schule. Im besten Falle betreuen sie angehende Lehrerinnen und Lehrer so, dass das Wissen und Können beider Orte für diese wirksam werden kann. Ein Team mit Vertreterinnen und Vertretern dreier Universitäten in Mecklenburg-Vorpommern (fünf Fachdidaktiken und Schulpädagogik) entwickelte, mit besonderem Blick für die Vernetzungen aller Ebenen des Lehrens und Lernens, das Lehrkonzept Mentor*innenqualifizierung $M-V$. Der vorliegende Text bietet erstens Einblicke in das in Form einer Design Based Research Studie über drei Zyklen hinweg entwickelte Lehrkonzept, er referiert zweitens zentrale Designprinzipien für die Entwicklung zukünftiger Qualifizierungsprogramme und entfaltet drittens das im Projektzeitraum entwickelte theoretische Modell des gegenstandsbezogenen Mentoring.

\section{Einführung}

Die Mentor*innenqualifizierung Mecklenburg-Vorpommern erwuchs ursprünglich aus dem Ziel, eine kompakte Schulung für Lehrerinnen und Lehrer zu entwickeln, die Studierende und Referendarinnen und Referendare in der Schulpraxis betreuen. Schon die ersten Ideen zu einem Curriculum und die zugehörigen Diskussionen in der Projektgruppe ${ }^{1}$ zeigten, dass sich der Blick weitete: Verschiedene Akteurinnen und Akteure mit ihren Interessen und verschiedene Fachdiskurse verlangten nach einer genaueren Betrachtung. Zugleich zeigte sich, dass

1 Lehrende der folgenden lehrerbildenden Institutionen sind im Projekt „MentorInnenqualifizierung M-V beteiligt: Hochschule für Musik und Theater Rostock (Fachdidaktik Musik), Universität Greifswald (Schulpädagogik im Fach Deutsch) sowie Universität Rostock (Projektleitung und Fachdidaktiken Biologie, Mathematik und Physik). Vgl. https://www.zlb.uni-rostock.de/qualitaetsoffensive-lehrerbildung/projektbereiche/mentorinnenqualifizierung/ (Abrufdatum: 29.11.2019) 
das Gesamtsystem Lehrerbildung auch als Ganzes betrachtet werden wollte. In der Folge entwickelte sich aus der Idee der kompakten, einjährigen Schulung ein umfassendes und theoretisch untermauertes Konzept einer Mentor*innenqualifizierung. In den Jahren der Entwicklung kam es sowohl zu theoretischen Weiterentwicklungen als auch zu einem fundierten Qualifizierungskonzept. Für die Entwicklung von Qualifizierungsangeboten für Mentorinnen und Mentoren an anderen Orten können diese Erkenntnisse nun genutzt, vertieft und weiterentwickelt werden.

Im Folgenden werfen wir einen Blick auf die Lernbegleitung in Praxisphasen durch Mentorinnen und Mentoren und der Mentor*innenqualifizierung (Kap. 2). Wir stellen Aspekte von Design Based Research, unserer Forschungsmethodik, vor und begründen deren Einsatz (Kap. 3). Anschließend stellen wir einen ersten theoretischen Aufschlag (Kap. 4) und das aktuelle Qualifizierungsprogramm (Kap. 5) vor. Danach führen wir die Leitprinzipien und die spezifischen Umsetzungsprinzipien des Qualifizierungsprogramms aus (Kap. 6). Zum Abschluss wird für einige der Designprinzipien die Umsetzung am Beispiel der fachspezifischen Qualifizierung Biologie erläutert (Kap. 7). Mit diesem Beitrag möchten wir die Mentor*innenqualifizierung M-V zur weiteren Diskussion stellen.

\section{Lernbegleitung entwickeln - Mentorinnen und Mentoren qualifizieren}

Die Begleitung von Studierenden in den studienbegleitenden Praxisphasen ist eine zentrale Aufgabe der Hochschulen im Lehramtsstudium. Die Studierenden und Dozierenden lernen und lehren in den Praxisphasen in „dritten Räumen“ (vgl. Zeichner 2010) zwischen Hochschule und Schule. Während an den Hochschulen die Verantwortlichkeiten für die bildungswissenschaftliche, fachdidaktische und fachliche Ausbildung in der ersten Phase geklärt sind, ist eine Arbeitsteilung bei der Lernbegleitung in den Praxisphasen weniger geregelt. Die formale Klärung von Verantwortlichkeiten zwischen Hochschulen und schulischem Lernort wird zwar versucht (vgl. Gröschner u.a. 2015), nach Gröschner u.a. (2015) sind die Fachdidaktiken für 25 Prozent der Praxisphasen allein und für noch einmal so viel zusammen mit den Bildungswissenschaften zuständig. Die Stärke der Praxisphasen liegt aber gerade auch in der Vernetzung des Wissens an Schule und Hochschule. Die Qualitäten der Lernbegleitung an sich und das Lernen der Lehramtsstudierenden sind damit jedoch noch nicht adressiert. Auch die Fragen, wer die Lernbegleiterinnen und Lernbegleiter in den Praxisphasen sind und wie diese bestmöglich in ihrer Arbeit zu unterstützen sind, damit sie das Lernen wirksam begleiten können, stehen erst seit kürzerer Zeit im Diskurs. 
In Mecklenburg-Vorpommern sind die Fachdidaktiken der Universitäten und Hochschulen für die schulpraktischen Übungen ${ }^{2}$ zuständig. Dabei betreuen entweder die Fachdidaktikerinnen und Fachdidaktiker selbst oder Lehrerinnen und Lehrer der Schulstandorte die Studierenden. Das Hauptpraktikum, welches die wesentlichen Unterrichtserfahrungen beinhaltet, wird von Dozierenden der Fachdidaktiken betreut. Im Rahmen des Projektes LEHREN in MV, gefördert durch die Qualitätsoffensive Lehrerbildung, stellte sich unsere Projektgruppe die Frage, wie die Qualität der Lernbegleitung in Praxisphasen gesteigert werden kann. Die Akteurinnen und Akteure fokussierten mit Schulpraktischen Übungen (Biologie- \& Physikdidaktiker), Hauptpraktikum (Mathematikdidaktik), dem neu geschaffenen Format Praxisjahr Schule (Musikpädagogik) oder schulpädagogischen Praxisseminaren (Schulpädagogik) auf unterschiedliche Praxisphasen. Im Projektverlauf zeigte sich neben der Bezugnahme auf bestehende Praktikumsformate, dass die Beteiligten auch neue Themengebiete in die Praxisphasenarbeit einbinden wollten. Beispielsweise wurde in schulpädagogischen Praxisseminaren (Universität Greifswald) die Einbindung offener, heterogenitätsorientierter Unterrichtsformen wie das Lernbüro ${ }^{3}$ anvisiert.

Die Biologiedidaktik widmete sich dem Transfer neuer Erkenntnisse aus dem Kompetenzbereich Erkenntnisgewinnung. Die Musikpädagogik fokussierte auf Fragen der unterschiedlichen Konzeptionierung von Kunst und künstlerischem Gestalten in Hochschule und Schule (Malmberg 2018a; Malmberg 2018b). Diese Themen konnten in den Bereichen allgemein-pädagogisches, fachdidaktisches und fachliches Wissen im Modell der professionellen Handlungskompetenz von Lehrerinnen und Lehrern (Baumert \& Kunter 2006) verortet werden. Die Anwendung dieses Wissens in den Unterrichtsversuchen der Praxisphasen und damit der Transfer konnte nicht als selbstverständlich hingenommen werden. Unsere Projektgruppe wählte die Qualifizierung von Lehrerinnen zu Mentorinnen und Lehrern zu Mentoren als Transfermöglichkeit.

Mentorinnen und Mentoren begleiten die individuelle Professionalisierung angehender Lehrerinnen und Lehrer im Überschneidungsraum zwischen den Ausbildungsinstitutionen (z.B. Hochschule) und dem Berufsfeld Schule.

2 In den Schulpraktischen Übungen betreut jeweils eine Lehrerin, ein Lehrer, eine Fachdidaktikerin oder ein Fachdidaktiker fünf Studierende. Jene halten semesterbegleitend, abwechselnd Unterrichtsstunden an einem Zeitpunkt in der Woche.

3 Das Lernbüro für das Fach Deutsch ist eine Form der Unterrichtsorganisation, die einen heterogenitätssensiblen Unterricht mit individualisierten Aufgaben vorsieht. Schülerinnen und Schüler können entlang von Bildungsstandards und mithilfe von Kompetenzrastern ihre eigenen Lernwege gestalten. Von den Betreuenden werden Lernaufgaben und Lernziele an die individuellen Möglichkeiten des einzelnen Kindes angepasst. Am Beispiel des Lernbüros können Lehrerinnen, Lehrer und Lehramtsstudierende die Umsetzung eines offenen, heterogenitätsorientierten und lernerzentrierten Unterrichts kennen lernen. 
Im Sinne dieser Definition beinhaltet eine Mentor*innenqualifizierung sowohl die Befähigung zur Lernbegleitung der angehenden Lehrerinnen und Lehrer als auch das Wissen um die Ausbildung. Insofern sind Mentorinnen und Mentoren weder reine Lernbegleiterinnen und Lernbegleiter, die fachunabhängig begleiten, noch sind sie reine Lehrpersonen der (Unterrichts-)Praxis. Hintergrundtheorien zum Lehren und Lernen sind für Lehrerinnen und Lehrer vorwiegend implizit verfügbar - Mentorinnen und Mentoren sollten diese im Sinne der Ausbildung explizieren können. Mentoring ist dann neben dem Unterrichten auch nicht nur eine weitere Aufgabe von Lehrerinnen und Lehrern.

Diese theoretischen Überlegungen zur Lernbegleitung und Ausbildung machte für das Qualifizierungsprogramm eine Struktur notwendig, in der sowohl überfachliche als auch fachliche Anteile (vgl. 5. in diesem Beitrag) enthalten sind. Die Diskussion, welche Kompetenzen die Mentorinnen und Mentoren erwerben sollten, orientierte sich einerseits an wissenschaftlichen Ergebnissen und andererseits an praktischen Erfahrungen im Kontext der Lehrerbildung und Weiterbildung. Zusätzlich mischte sich in die Diskussion immer wieder die Frage danach, welche Kompetenzen Studierende oder sogar Schülerinnen und Schüler erwerben können sollen, wenn wir diese oder jene Mentor*innenqualifizierung anbieten. In diesem Punkt zeigte sich das zentrale Desiderat: Ein theoretisches Modell fehlte, das Mentoring in seinen Wirkungen bis zur Lernsituation der Schülerinnen und Schüler hin abzubilden vermochte.

Für den Teil der überfachlichen Qualifizierung wurde einerseits an die Modelle und empirischen Ergebnissen zur Gesprächsführung (Kreis \& Staub 2013; Kreis 2012; Niggli 2005) und an die aktuellen Entwicklungen im Bereich der Forschung (Schnebel 2018) angeknüpft. Andererseits trugen Expertinnen und Experten der Betreuungspraxis eine Vielzahl an Best-Practice-Hinweisen bei (vgl. Malmberg \& Lion 2014). Die fachspezifischen Qualifizierungsteile knüpften ebenfalls an wissenschaftliche Ergebnisse und praxisbezogene Erfahrungen an. Für die Entwicklung und Weiterentwicklung stellte sich in der Folge die Frage, welche Qualitäten das Programm haben sollte und wie sich die Qualitätsentwicklung des Programms eng an die Theoriearbeit anbinden ließe. Der Forschungsansatz der Entwicklungsforschung bot uns das geeignete Instrumentarium.

\section{Entwicklungsforschung/Design Based Research (DBR)}

In den letzten zehn bis fünfzehn Jahren etablierte sich innerhalb der Lehr-/Lernforschung im deutschsprachigen Raum das Forschungsparadigma der Entwicklungsforschung. Das Grundziel von Entwicklungsforschung, nämlich innovative Konzepte für die unterrichtliche Zukunft bereitstellen zu wollen, erklärt Arthur Bakker sehr pointiert: 
Most educational research describes or evaluates education as it currently is. Some educational research analyzes education as it was. Design research, however, is about education as it could be or even as it should be (Bakker 2019, 3, Hervorhebungen v. Bakker).

Entwicklungsforschungsprojekte treiben die Innovation von in der Zukunft stimmiger (Unterrichts-)Praxis und die dazugehörige Theoriebildung voran (van den Akker u.a., 2006; Cobb u.a. 2003). Es wird theoriebasiert an einem (fach-)didaktischen Problem gearbeitet, für das - zunächst als normative Setzung (Design) - ein innovatives Unterrichtskonzept entwickelt wird. Dieses Konzept wird in mehreren Zyklen erprobt, es wird - empiriegestützt-evaluativ vorgehend - weiterentwickelt und immer enger an kontextuelle Erfordernisse und gewünschte Qualitäten angepasst (Re-Designs), um am Ende als Entwicklungsprodukt vorzuliegen. Daneben entsteht Theoriewissen zum unterrichtlichen Problem. Es handelt sich bei diesem Theoriewissen vor allem um sogenannte actionable knowledge, also Wissen, das dem besseren Verstehen der Lernvorgänge dient. Beide Ebenen - die Entwicklung des Unterrichtskonzeptes und die Verfeinerung des Theoriewissens - schreiten laufend verschränkt voran. ${ }^{4}$

\subsection{DBR: Der Konzeptentwicklung Zeit geben}

War zu Beginn des Projektes (ein Jahr vor Beginn des ersten Qualifizierungsdurchlaufs) noch ein Kontrollgruppendesign geplant, stellte sich rasch heraus, dass die Gruppen zu klein sein würden, um ausreichend belastbare Evaluationsergebnisse zu erzielen. Es erschien uns darüber hinaus äußerst attraktiv, in einem so jungen didaktischen Feld wie der Qualifizierung von Mentorinnen und Mentoren nicht nur zu messen und zu bewerten, was wir als Ergebnisse unseres Programmes festmachen können würden, sondern dem Entwicklungsprozess des Konzeptes über mehrere Jahre und Kohorten hinweg Zeit zu geben, also das Design mehrfach entlang unserer Erkenntnisse zu re-designen. Wir wollten das Konzept längerfristig entwickeln und mögliche Wirkungen ausreichend im Blick behalten, um so zu einem qualitativ belastbaren Lehrkonzept zu gelangen, damit wir fundiert argumentieren können.

Die Konzeptentwicklung stand dabei in dauerndem Diskurs mit der während des Projektes laufend anwachsenden theoretischen Literatur zum Mentoring sowie

4 Verschiedene Bezeichnungen existieren für diesen Forschungsstil nebeneinander: fachdidaktische Entwicklungsforschung (Prediger 2012), entwicklungsorientierte Bildungsforschung (Reinmann 2015) und - weil frühe Projekte vor allem aus Amerika und den Niederlanden stammen - auch die englischen Begriffe Design Research (McKenney \& Reeves 2012), Design Research in Education (Bakker 2019), Educational Design Research (Cobb 2003, Van den Akker 2013) oder Design Based Research, kurz DBR (Euler \& Sloane 2014; Reinmann 2018). In unseren folgenden Darstellungen nutzen wir den Begriff Design Based Research (DBR), um auch zum englischsprachigen Diskurs anschlussfähig zu bleiben. 
den empirischen Auswertungen unserer Daten im Projekt. McKenney \& Reeves veröffentlichten 2012 (S. 77) eine anschauliche Darstellung zu diesem Vorgehen (Abb. 1):

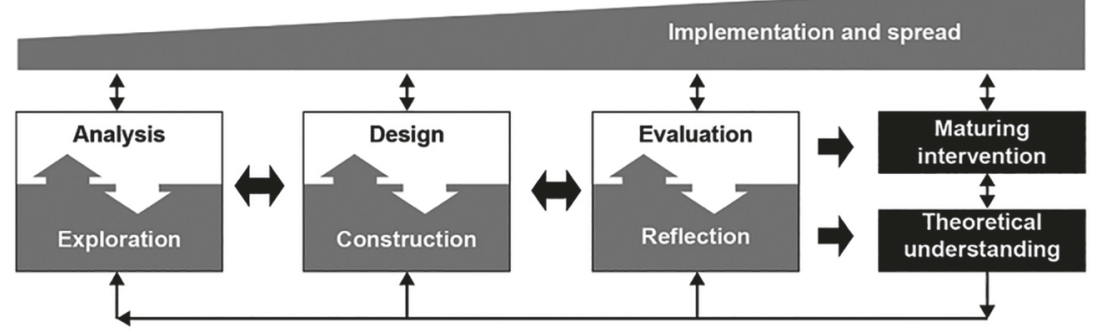

Abb. 1: Generisches Modell Design Research im Bildungskontext (McKenney \& Reeves 2012)

Sehr schön sichtbar ist in Abb. 1 die laufende Vernetztheit von Praxisentwicklung (die auf der oberen Ebene zur Maturing Intervention führt) mit der Theoriearbeit (die auf der unteren Ebene das Theoretical Understanding vertieft) unter Zuhilfenahme der Evaluation erhobener Daten.

\subsection{Ausgangspunkte für Design und Re-Designs}

Das Anfangsdesign der Kohorte 17/18 und die beiden Re-Designs 18/19 und 19/20 wurden auf Basis verschiedener im Prozess erstellter Dokumente sowie durch begleitende Evaluationen auf der Basis empirischer Daten und Materialien entwickelt. Es handelt sich um vier Kategorien von Quellen:

Erstens wertete die Projektgruppe theoretische Literatur aus. Ihre Aufbereitung und Diskussion wurde regelmäßig miteinbezogen, beispielsweise Erkenntnisse von Publikationen zur Professionalisierung (Baumer \& Kunter 2006), zum Mentoring (Niggli, 2005; Kreis, 2012) und zur Praxisphasengestaltung. Sie wurden in Form von Lektüre, Exzerpten oder Präsentationen ins Projektteam geholt. Zudem fanden Anpassungen, Erweiterungen oder Weiterentwicklungen dieser theoretischen Grundlagen statt.

Zweitens wurden praxisorientierte Dokumente, die normative Setzungen vornehmen, aus den Bereichen Schulunterricht, Studium und Lehrerinnenbildung einbezogen: Übersichten zu den angestrebten Kompetenzen im Unterricht, im Lehramtsstudium oder für Mentorinnen und Mentoren. Zu diesen Dokumenten gehören auch Handreichungen zum Schreiben eines Unterrichtsentwurfes oder mögliche Fragen für Unterrichtsbesprechungen. 
Drittens wurden Dokumente, die bei der Organisation des Projektprozesses entstanden sind, laufend ausgewertet: Der Projektantrag, Zwischenberichte zum Projektstand oder Teilnahmeübersichten. Besonders prägend sind die Teambesprechungen mit ihren Entscheidungen, die als Sitzungsprotokolle vorliegen. Design-Entstehung und Gründe für Re-Designs sind dadurch sehr gut nachvollziehbar.

\subsection{Auswertungen empirischer Daten}

Viertens wurden quantitative und qualitative Daten der begleitenden Teilnehmer-Evaluationen einbezogen. Die Mentorinnen und Mentoren beurteilten die Qualifizierung insgesamt bereits im ersten Jahrgang auf einer 9-stufigen Likert-Skala ( $\mathrm{n}=24,9$ Items) mit Mittelwerten von 7,1 (,Die Inhalte der Veranstaltung waren geeignet, meine eigene Unterrichtspraxis zu bereichern“) bis 8,4 („Der Austausch mit Kolleg*innen war ein wertvoller Teil der Qualifizierung."). Ein detaillierter Blick auf einzelne Qualifizierungsabschnitte zeigte, dass die fachspezifischen Module von den Mentorinnen und Mentoren als ein wenig besser eingeschätzt wurden. Diese Ergebnisse flossen zusammen mit den offenen Fragen der Evaluation im großen Maße in die Re-Designs ein. (Beispielfrage: „Was waren aus Ihrer Sicht die drei wichtigsten Dinge, die Sie in den allgemeinen Modulen gelernt haben?").

Mit der Frage nach der Bedeutung einzelner Weiterbildungsinhalte (11 Items) der Mentor*innenqualifizierung konnten die Ergebnisse von Schüpbach (2005) sowohl für den Beginn als auch zum Abschluss des ersten Qualifizierungsjahrganges repliziert werden. Die Lehrerinnen und Lehrer schätzten in fast allen Bereichen (z.B. Unterrichtsbesprechung, Gesprächsführung) die Bedeutung der Weiterbildungsinhalte ähnlich ein, wie die Lehrerinnen und Lehrer in der Studie von Schüpbach (2005). Die Vertiefung der fachdidaktischen Kenntnisse werteten unsere Lehrerinnen und Lehrer etwas höher. Dies mag mit der höheren Präsenz der Fachdidaktiken zusammenhängen - auf personaler Ebene (Leitungsteam) und auf inhaltlicher Ebene (fachdidaktische Anteile des Qualifizierungsprogrammes). Zusätzlich erhoben einzelne Fachbereiche im Zusammenhang mit den Qualifizierungsarbeiten weitere Daten. Beispielsweise wurde in der Biologiedidaktik zur Einordnung der Praxisphasen die Kompetenzentwicklung der Studierenden (Gröschner \& Schmitt, 2009) über alle Lehrveranstaltungen erfragt (Nestler \& Retzlaff-Fürst i.V. b). 


\section{Lernbegleitungen ebenenübergreifend verknüpfen}

Die bisherigen Forschungsarbeiten zum Mentoring fokussieren besonders auf die Kompetenz zur Gesprächsführung (Kreis, 2012) oder Gesprächsebenen und -arten (Niggli 2005). Auf die Fachspezifität des Mentorings wird mit dem Terminus „fachspezifisch-pädagogisches Unterrichtscoaching" bei Staub \& Kreis (2013) verwiesen. Durch den Verweis auf Fachwissen und fachdidaktisches Wissen stützt auch das Modell der professionellen Handlungskompetenz (Baumert \& Kunter 2006) diese Ausrichtung.

Schnebel (2018) fordert jüngst, dass die Entwicklung einer Mentor*innenqualifizierung an den Kompetenzen der Studierenden ansetzen müsse. Diese Kompetenzbeschreibungen beziehen sich oft auf das Unterrichten. Eine Mentor*innenqualifizierung adressiert somit ein Mehrebenensystem, das die Qualifizierung selbst, die Entwicklung der Studierendenkompetenzen in den Praxisphasen und das Lernen der Schülerinnen und Schüler im schulischen Unterricht einbezieht.

Ähnliche Überlegungen zu einem Mehrebenensystem (hier für die gegenstandsbezogene Ausbildung von Multiplikatorinnen und Multiplikatoren) führten Prediger u.a. (2017) zur Modellierung des sogenannten 3Tetraedermodell zur gegenstandsbezogenen Professionalisierung. Für die Beschreibung der Zusammenhänge einer Mentor*innenqualifizierung ist dieses Modell sehr nützlich (Abb. 2; vgl. auch Nestler \& Retzlaff-Fürst i.V. a).

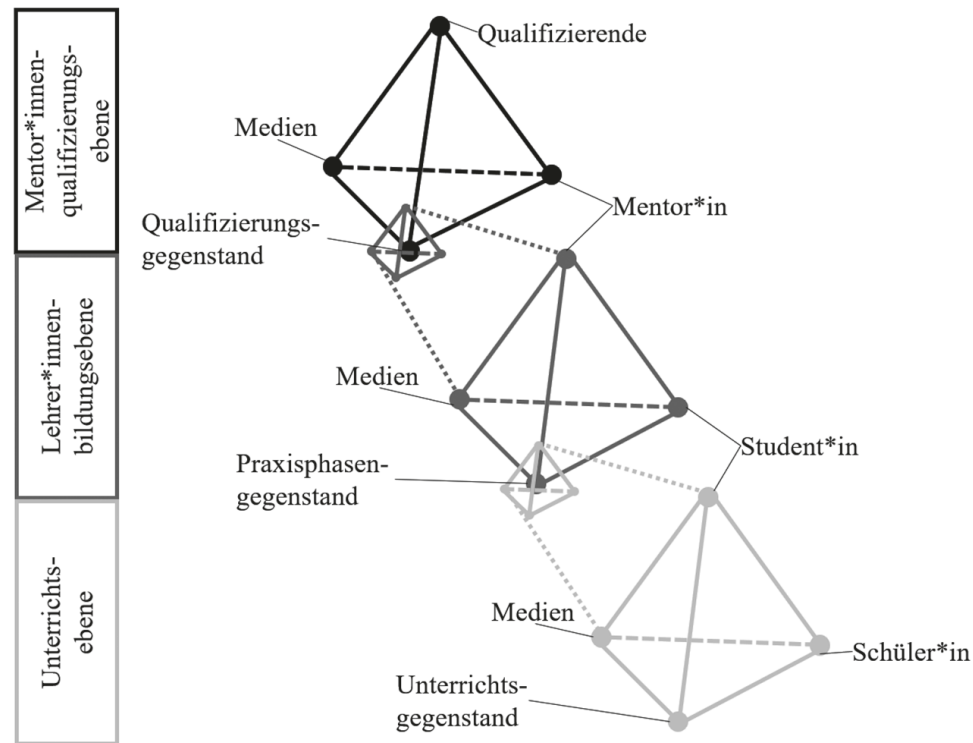

Abb. 2: Lernbegleitung im Tetraedermodell zum gegenstandsbezogenen Mentoring 
Jeder Tetraeder steht dabei für eine Lernebene, in der Lernbegleitende und Lernende in Auseinandersetzung mit einem Gegenstand und unter der Verwendung spezifischer Materialien und Medien Lernsituationen gestalten (Abb. 2). Der Gegenstand der Mentor*innenqualifizierung ist die Praxisphase (Abb. 2, dunkelgrau). Die Praxisphase hat als Gegenstand den zu planenden, durchzuführenden und zu evaluierenden Unterricht (Abb. 2, hellgrau).

Folgt man Abb. 2 müssen für die Gestaltung einer Mentor*innenqualifizierung alle drei Ebenen von Lernsituationen gedanklich mit einbezogen werden: die Qualifizierung, die Lehrerbildung und der schulische Unterricht. Die Lernbegleiterinnen und Lernbegleiter, Mentorinnen und Mentoren und Studierende sind in diesem Modell zugleich Lehrende und Lernende.

Beispielsweise dienen die Merkmale der Unterrichtsqualität (Meyer 2018, Helmke 2017) zur Reflexion der Unterrichtsqualität. Somit werden Kriterien für die Ausgestaltung der Lernsituation Unterricht gegeben. Mentorinnen und Mentoren nutzen die Qualitätsmerkmale oder abgeleitete Materialien als papierne Medien. Sie dienen somit zur Gestaltung der Lernsituation. Idealerweise kennen die Studierenden diese Qualitätsmerkmale schon über universitäre Seminare. In den Unterrichtsbesprechungen können sich Mentorinnen und Mentoren und Studierende auf diese beziehen. Die gezielte Anwendung der Unterrichtsqualitätsmerkmale können wiederum Gegenstand der Mentor*innenqualifizierung sein. Insofern wirkt die Mentor*innenqualifizierung bis zur Unterrichtsebene, weil die Mentorinnen und Mentoren die Qualitätsmerkmale mediengestützt einbringen, anwenden und reflektieren können.

Viele andere Aspekte, wie den Einbezug von Alltagsvorstellungen (Abb. 3, B) im Biologieunterricht oder die Förderung des Klassenklimas (Abb. 3, A), können Mentorinnen und Mentoren schwieriger theoriegestützt in die Praxisphase einbringen, obwohl sie auch Gegenstand der Praxisphase sein könnten (Abb. 3, $\mathrm{C}$ links). Ihnen fehlen dafür die Medien oder Kenntnisse. Die genannten Ziele sind aber sehr wohl der Gegenstand universitärer Lehrveranstaltungen (Abb. 3, $\mathrm{C}$ rechts). In den Praxisphasen erfolgt die Wahl der Gegenstände nur in einigen Fällen systematisch. Viel häufiger findet sich ein chronologisches Vorgehen durch den Unterrichtsentwurf oder die beliebige Wahl nach dem, „was beobachtet wurde" (vgl. auch Beckmann et al. in diesem Band). Einen Weg zur Wahl der Gegenstände der Praxisphasen finden wir in diesem Band, bspw. stellen Hesse und Lütgert den Einsatz des Unterrichtsdiagnoseinstruments EMU (Helmke u.a. 2018) dar. 


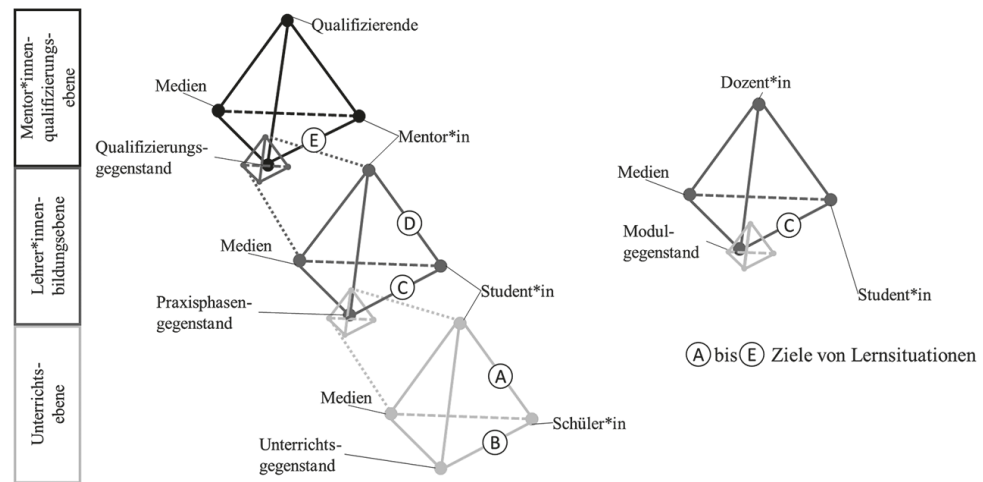

Abb. 3: Ziele von Lernsituationen im Tetraedermodell zum gegenstandsbezogenen Mentoring

Auf der anderen Seite fokussieren viele Konzepte die Beziehung von Mentorin und Mentor mit Studierenden (Abb. 3, D) als Gegenstand der Mentor*innenqualifizierung (Abb. 3, E). In der Folge sind zwei Phänomene beobachtbar:

Der Transfer des Wissens aus der universitären Veranstaltung in die Praxisphase erfolgt über die Studierenden (Abb. 4, 1). Bei der Fülle an universitären Veranstaltungen und den hohen Anforderungen, die sich für die Studierenden bei den ersten Unterrichtsversuchen stellen, ist die Wirkung dieser Kette fraglich. Die Qualifizierung der Mentorinnen und Mentoren wird, wenn es sie gibt, mit Blick auf die Gesprächsführung und Beziehung in der Praxisphase geplant (Abb. 4, 2). Die Auseinandersetzung mit den Gegenständen der Praxisphase ist in diesem Fall kein umfangreicher Gegenstand der Qualifizierung der Mentorinnen und Mentoren.

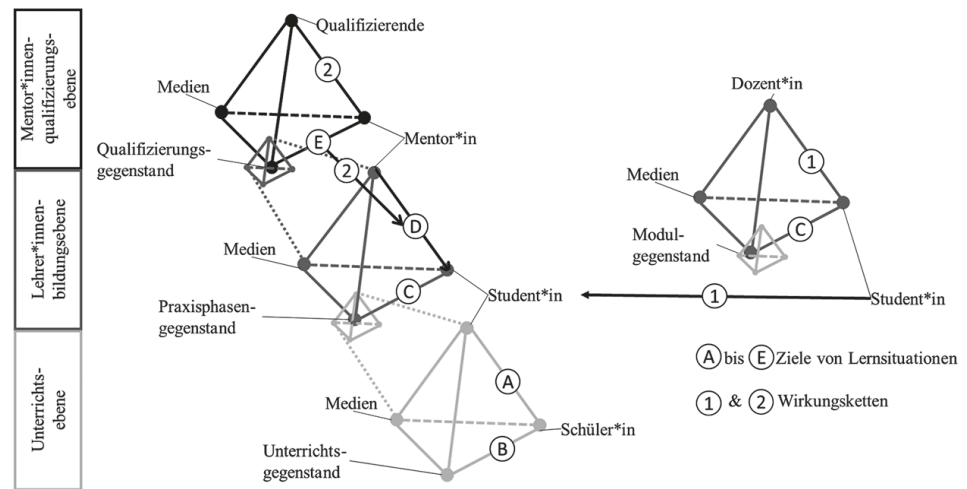

Abb. 4: Wirkungsketten in Lernsituationen im Tetraedermodell zum gegenstandsbezogenen Mentoring 
Die Kompetenzentwicklung der Studierenden wird aber gerade durch die Auseinandersetzung mit den Gegenständen der Praxisphase erreicht. Die beiden Wirkungsketten (Abb. 4, 1 \& 2) enden in diesen Fällen in der Praxisphase. Durch die Mentor*innenqualifizierung erfolgt dabei keine Adressierung der Gegenstände der Praxisphase (Abb. 4, C). In einigen Fällen sind dann den Mentorinnen und Mentoren Forschungsergebnisse, Modelle und Theorien weder als Wissen noch als Medium verfügbar. Für die Lernsituation Praxisphase bedeutet dies, dass die Mentorinnen und Mentoren entweder auf ihre eigenen Theorien zurückgreifen müssen oder die Aufgabe des Einbringens aktuellen Wissens durch die Studierenden erfüllt werden muss. Dies kann sowohl Mentorinnen und Mentoren als auch Mentee überfordern.

\section{Das Konzept Mentor*innenqualifizierung M-V}

Die folgende Tabelle 1 zeigt den Ablauf der Mentor*innenqualifizierung M-V in seiner aktuellen Fassung (Studienjahr 2019/20, 3. Kohorte). Weiße Tabellenzeilen enthalten die fachübergreifenden Qualifizierungsanteile, grau unterlegte Tabellenzeilen zeigen ein Umsetzungsbeispiel eines gegenstandsbezogenen Anteils (hier: Biologie). Die Qualifizierung geht über ein Jahr und umfasst 90 Stunden, davon 45 Präsenzstunden und 45 Stunden Selbststudium. Ungefähr die Hälfte der Zeit gehört der fachübergreifenden, die andere Hälfte der gegenstandsbezogenen Qualifizierung. Mentorinnen und Mentoren, die die Qualifizierung durchlaufen, betreuen im Normalfall parallel dazu bereits Studierende in einem der Praktikumsformate. Immer wieder werden daher Verbindungen zwischen der Qualifizierung und der konkreten Betreuungsarbeit gezogen (z.B. in Reflexionen oder Aufgaben, die in der Praxis zu lösen sind und danach wieder in die Qualifizierung mitgebracht werden). Tabelle 1 zeigt die Programmteile über ein Jahr. 
Tab. 1: Zeitlauf und Inhalte eines Jahres Mentor*innenqualifizierung-MV

\begin{tabular}{|c|c|}
\hline Zeitpunkt + Thema & Inhalte \\
\hline $\begin{array}{l}\text { September } \\
\text { Allgemeine Qualifizierung - } \\
\text { Einführung } 1 \\
(1 \text { Tag) }\end{array}$ & $\begin{array}{l}\text { Die eigene Professionalisierung und die der Mentee sind die Aus- } \\
\text { gangspunkte der Mentor*innenqualifizierung. Die Kompetenzentwick- } \\
\text { lung der Studierenden in den verschiedenen Praxisphasen wird in den } \\
\text { Blick genommen. Der Qualifizierungstag endet mit der Einführung und } \\
\text { Übungen zur ko-konstruktiven Gesprächsführung nach Kreis (2012). }\end{array}$ \\
\hline $\begin{array}{l}\text { September } \\
\text { Allgemeine Qualifizierung - } \\
\text { Einführung } 2 \\
(1 \text { Tag) }\end{array}$ & $\begin{array}{l}\text { Am zweiten Qualifizierungstag wird die Mentorinnen- und Mento- } \\
\text { renrolle zwischen Schule und Universität diskutiert. Ausgehend von } \\
\text { Einführungen zum konstruktivistischen Lernen wird der Bogen zur } \\
\text { Betreuung individuell unterschiedlich lernender Mentees gelegt. Mit } \\
\text { weiteren Übungen zur Gesprächsführung und Unterrichtsvor- und } \\
\text {-nachbesprechungen werden die einführenden Qualifizierungstage } \\
\text { abgeschlossen. }\end{array}$ \\
\hline $\begin{array}{l}\text { Januar } \\
\text { Allgemeine Qualifizierung - } \\
\text { Reflexionstreffen } \\
\text { (2 Nachmittage) }\end{array}$ & $\begin{array}{l}\text { Die Mentorinnen und Mentoren betreuten während des laufenden } \\
\text { Wintersemesters bereits Studierende und Referendarinnen und } \\
\text { Referendare. In den Reflexionstreffen im Januar werden zentrale } \\
\text { Inhalte der Einführungen aufgegriffen und vor dem Hintergrund der } \\
\text { Betreuungserfahrungen ausgewertet und diskutiert. }\end{array}$ \\
\hline $\begin{array}{l}\text { Februar } \\
\text { Zukunftswerkstatt „Quali- } \\
\text { tätsverständnis Mentoring“ } \\
\text { (2 Tage) }\end{array}$ & $\begin{array}{l}\text { Mit Mentorinnen und Mentoren aus schon länger bestehenden } \\
\text { professionellen Lerngruppen, und mit weiteren Akteurinnen und } \\
\text { Akteuren wie Studierenden, Referendarinnen und Referendaren und } \\
\text { Fachseminarleiterinnen, wird das gemeinsame Verständnis von gutem } \\
\text { Mentoring weiterentwickelt. }\end{array}$ \\
\hline $\begin{array}{l}\text { Januar } \\
\text { Fachspezifische Qualifizie- } \\
\text { rungen - Reflexionstreffen } \\
\text { (1 Nachmittag) }\end{array}$ & $\begin{array}{l}\text { Die Betreuung der Schulpraktischen Übungen schafft gegenstands- } \\
\text { bezogene Bedarfe der Mentorinnen und Mentoren, diese werden } \\
\text { inhaltlich vermittelt, gemeinsam reflektiert, vor den Theorien und } \\
\text { Erfahrungen diskutiert und in Teilen in späteren Veranstaltungen } \\
\text { aufgenommen. (Bsp. Biologie) }\end{array}$ \\
\hline $\begin{array}{l}\text { Februar } \\
\text { Gemeinsame fachspezifi- } \\
\text { sche Qualifizierung } \\
\text { (1 Tag) }\end{array}$ & $\begin{array}{l}\text { Dieser gemeinsame Qualifizierungstag stellt eine Brücke } \\
\text { zwischen dem allgemeinen und fachspezifischen Teil der } \\
\text { Mentor*innenqualifizierung dar. Die Mentorinnen und Mentoren setzen } \\
\text { sich in fächerspezifischen Gruppen mit dem Ablauf des Studiums } \\
\text { im Fach und einem wichtigen Praxisphasengegenstand des eigenen } \\
\text { Faches auseinander. Die Erkenntnisse werden im Plenum diskutiert. }\end{array}$ \\
\hline $\begin{array}{l}\text { März } \\
\text { Fachspezifische Qualifizie- } \\
\text { rungen } \\
\text { (1 Tag) }\end{array}$ & $\begin{array}{l}\text { Das Feedback zu Unterrichtsentwürfen ist eine wichtige Aufgabe von } \\
\text { Mentorinnen, Mentoren und Dozierenden. An diesem Weiterbildungs- } \\
\text { tag werden Wege gezeigt und gemeinsam diskutiert schriftlich und } \\
\text { mündlich zielführendes Feedback zu geben. (Bsp. Biologie) }\end{array}$ \\
\hline $\begin{array}{l}\text { März } \\
\text { Fachspezifische Qualifizie- } \\
\text { rungen } \\
\text { (1 Tag) }\end{array}$ & $\begin{array}{l}\text { Mit Evolution und dem Kompetenzbereich Bewerten werden aktuelle } \\
\text { Themen aus Fachwissenschaft und Fachdidaktik diskutiert. Übungen } \\
\text { zu Unterrichtsbesprechungen spannen den Bogen zur allgemeinen } \\
\text { Qualifizierung. (Bsp. Biologie) }\end{array}$ \\
\hline $\begin{array}{l}\text { Juni } \\
\text { Wissenschaftstagung } \\
(1 \text { Tag) }\end{array}$ & $\begin{array}{l}\text { Die wissenschaftliche Entwicklung der Lernbegleitung von Studie- } \\
\text { renden soll fortwährend in die Qualifizierung einfließen. Aus diesem } \\
\text { Grund diskutiert die Wissenschaft an diesem Tag aktuelle Befunde. } \\
\text { Der Besuch durch die Mentorinnen und Mentoren ist freiwillig. }\end{array}$ \\
\hline $\begin{array}{l}\text { Juni } \\
\text { "Tag des Mentoring“ } \\
\text { (1 Tag) }\end{array}$ & $\begin{array}{l}\text { Die Diskussion der beteiligten Akteurinnen und Akteure ist ein } \\
\text { Qualiätsmerkmal der Mentor*innenqualifizierung. Aus diesem Grund } \\
\text { diskutieren Mentorinnen, Mentoren, Wissenschaftlerinnen und Wis- } \\
\text { senschaftler gemeinsam die Weiterentwicklung des Themas. }\end{array}$ \\
\hline
\end{tabular}




\section{Designprinzipien}

DBR Projekte nutzen unterschiedliche Formen, um ihre Entwicklungsprodukte und theoretischen Erkenntnisse für andere nachvollziehbar und - ein wichtiges Ziel von DBR - auch in deren Kontexte übertragbar zu machen (vgl. spread in Abb.1). Eine dafür gut geeignete Darstellungsform sind Designprinzipien. Diese können als „Kristallisationspunkte“ (Euler 2014b, 97) oder als „die Essenz des Entwicklungsprozesses“ (Malmberg i.V.) gesehen werden. Sie bezeichnen hochkonzentriert die zentralen Qualitäten eines Unterrichtkonzeptes und erleichtern damit die Übertragbarkeit in andere Kontexte. Sie bieten den Lehrenden eine normative Basis für ihr Handeln, „they provide guidance and direction, but do not give ,certainties' (Plomp 2007, 22)" und sie besitzen einen mittleren Grad an Generalisierung. Man kann sie als ein Produkt der Mitte zwischen Theorie und Unterrichtspraxis sehen. Sie „bilden eine Brücke zwischen den Ansprüchen der wissenschaftlichen Erkenntnisgewinnung und der didaktischen Praxisgestaltung " (Euler 2014a, 105).

\subsection{Entwicklungsprinzipien im Anfangsdesign}

In unserer Projektgruppe stand zu Beginn eine ausführliche Klärung unserer Vorstellungen von gutem Mentoring (normative Ebene). Ausgehend vom Bedingungsfeld wurde mithilfe der in Kap. 3 beschriebenen Entwicklungsforschungsstrategien im Projektteam ein geteiltes Verständnis von Mentoring festgelegt (vgl. Malmberg, Nestler \& Piotraschke 2018, 47-48). Diese Entwicklungsprinzipien grundierten das Anfangsdesign des Lehrgangs (1. Kohorte) mit a) inhaltlichen Ausrichtungen und b) strukturellen Ausrichtungen.

Folgende Entwicklungsprinzipien waren Konsens:

a) inhaltlich sollten die Mentorinnen und Mentoren:

- implizites Professionswissen in Reflexionsgesprächen explizit machen lernen,

- eine stärkenorientierte Beobachtungs- und Rückmeldehaltung erwerben,

- eine ko-konstruktive Gesprächskultur verinnerlichen und anwenden können,

- ein professionelles Selbst als Mentor bzw. Mentorin mit gestärkter und aktueller fachlicher und fachdidaktischer Expertise entwickeln sowie

- erworbene Fähigkeiten in den Domänen der Fächer und ihrer Kulturen umsetzen.

b) strukturell erschien wichtig:

- Die Mentorinnen und Mentoren werden als brückenbildend zwischen Schule und Hochschule gesehen; unter ihrer Einbindung wird versucht, den dritten Raum zu stärken. 
- Mittelfristig werden professionelle Lerngruppen in den Fächern angepeilt.

- Die Qualifizierung wird in Balance von teilhabezentrierten Vermittlungsformen (schon vorhandene Expertisen werden bewusst gemacht, genutzt und ausgetauscht) sowie Instruktion gestaltet.

Die Festlegungen waren wichtig, um Kohärenz im Gesamtlehrgang zu erreichen. Es handelte sich um ein Team aus fünf Fachdidaktiken und der Schulpädagogik, das zwar die Hälfte der Qualifizierung (fachübergreifender Teil) gemeinsam verantwortete, daneben jedoch jeweils eigenständig gegenstandsbezogene Qualifizierungsveranstaltungen gestaltete. Aus den Entwicklungsprinzipien heraus verständigten wir uns anschließend auf Teilzielkompetenzen, die die Detailplanung und die laufenden Evaluationen lenken sollten. In den jeweiligen Fächern wurden darüber hinaus eigenständig gegenstandsbezogene Kompetenzen für das Mentoring formuliert.

\subsection{Designprinzipien für die Entwicklung zukünftiger Programme}

Nach Durchlaufen dreier Zyklen und sämtlicher Auswertungen erscheinen uns die folgenden Designprinzipien für die Erstellung von Qualifizierungsprogrammen für Mentorinnen und Mentoren zentral. Einige der Entwicklungsprinzipien aus 6.1. haben sich erkennbar weiterentwickelt, neue sind hinzugetreten. Die folgenden 14 Designprinzipien können als Handlungsmuster für die Entwicklung ähnlicher Lehrkonzepte genutzt werden.

\subsubsection{Leitprinzipien}

Leitprinzipien meinen pädagogische Ausrichtungen, die das Lehrkonzept eher grundsätzlich leiten. Sie sind meist gut in der Theorie verankert und werden oft auch von dort hergeleitet (Van den Akker 1999). ${ }^{5}$ Das erste Leitprinzip erscheint uns als Metaprinzip, das in viele weitere Prinzipien hineinreicht:

\section{Meta-Designprinzip: MQ adressiert die ebenenübergreifenden Wirkungsketten.}

Mentorinnen und Mentoren stehen mit ihrer Arbeit in weitreichenden Wirkungszusammenhängen (vgl. Kap. 4). Aktuelles Wissen aus den Fachdisziplinen und Fachdidaktiken erreicht sie über die Studierenden. Im Idealfall gehen sie mit diesem Wissen so um, dass sie einerseits das Fachlernen der Mentees und andererseits das ihrer Schülerinnen und Schüler gut unterstützen. Sie unterstützen auf diese Weise optimal die „Wirkungsketten“.

5 Van den Akker (1999) unterscheidet substantive design principles (Leitprinzipien) von procedural design principles (Umsetzungsprinzipien). 


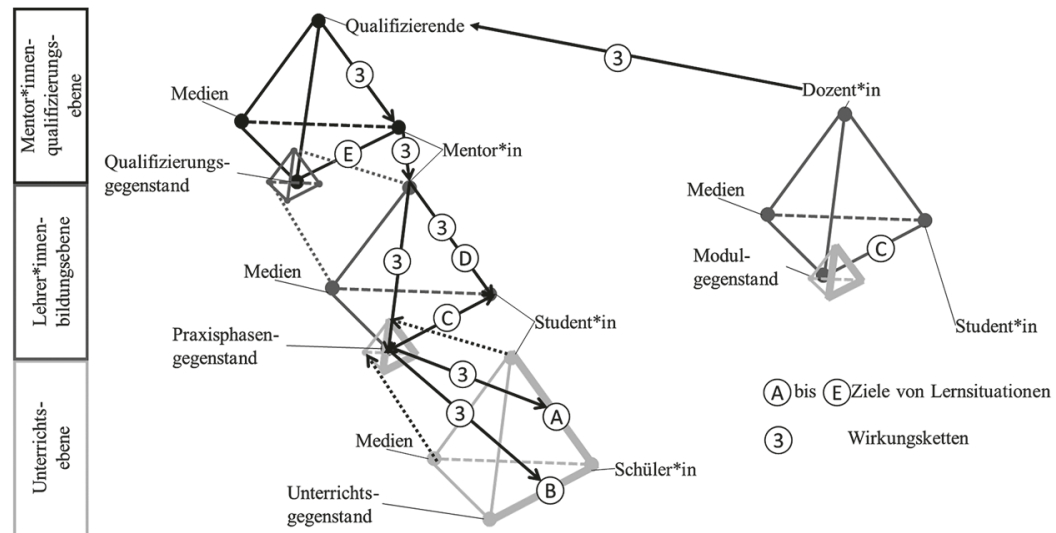

Abb. 5: Wirkungsketten im Tetraedermodell zum gegenstandsbezogenen Mentoring

Eine Qualifizierung für Mentorinnen und Mentoren sollte Wirkungsketten möglichst über alle drei Ebenen des Tetraedermodells zum gegenstandsbezogenen Mentoring (Abb. 5, 3) adressieren. Die universitäre Ausbildung hofft dann nicht auf einen Transfer durch die Studierenden, sondern ermöglicht es, dass Mentorinnen und Mentoren die Praxisphase als Lernsituation mit Bezug zu den Gegenständen der Ausbildung gestalten können.

In der konkreten Arbeit sind viele Spielarten denkbar. Bspw. können Fachthemenschwerpunkte gesetzt werden, die sowohl Mentees in universitären Seminaren als auch Mentorinnen und Mentoren in ihren professionellen Lerngruppen (z.B. in Kooperation mit Fachwissenschaften) über einen Zeitraum gleichzeitig bearbeiten, damit sie dann im Praktikum „vom Gleichen sprechen“ (vgl. Nestler \& Retzlaff-Fürst, i.V. a). Oder es wird ein neues pädagogisches Konzept gleichzeitig für Mentorinnen bzw. Mentoren mit ihren Mentees an den Schulen eingeführt. Dieses Adressieren der ebenenübergreifenden Wirkungsketten definieren wir als übergeordnetes Prinzip.

\section{Die Fachdidaktiken moderieren herrschende Fachlichkeitskonstruktionen, Mento-} rinnen und Mentoren werden dabei als Teil des Fachdidaktik-Teams verstanden.

Praxisphasen können als Orte gesehen werden, an denen ganz unterschiedliche Konstruktionen der Fachlichkeit geradezu krisenhaft aufeinandertreffen. So existieren für jedes Schulfach „Schulfachlogiken“ neben Disziplinenlogiken aus wissenschaftlichen Fächern, neben Konstruktionen aus der Gesellschaft oder subjektiven Fachlichkeitskonstruktionen (Martens u.a. 2018). Häufig unterscheiden sich diese Konstruktionen, sie werden bisweilen sogar als sich stark unterscheidende Konzepte gesehen. Bislang waren Studierende in der Bezugnahme dieser unterschiedlichen Logiken alleingelassen. 
Fachdidaktiken sind im Idealfall Orte der Moderation der Fachlichkeitskonstruktionen (Malmberg, i.V.). In der Mentor*innenqualifizierung wiederum sollten Fachlichkeitskonstruktionen ausreichend thematisiert und offengelegt werden, damit Mentorinnen und Mentoren die Entwicklung stimmiger Fachlichkeitskonzepte ihrer Studierenden gut begleiten können. Unserer Erfahrung nach gelingt dies leichter, wenn die Mentorinnen und Mentoren sich (auch) als Teil des hochschulischen Fachdidaktik-Teams verstehen können und in dortige Diskurse eingebunden werden.

3. MQ bezieht alle beteiligten Akteurinnen und Akteure ausreichend ein, um geeignete Strukturen zu schaffen: Mentorinnen und Mentoren, Mentees, Fachdidaktiken, Schulleitungen, Schulamt, IQMV (2. und 3. Phase) und Fachwissenschaften.

Hürden, die sich während der Entwicklung und Durchführung der Mentor*innenqualifizierung stellen, können durch laufende Kooperation mit unterschiedlichen Akteurinnen und Akteuren deutlich verkleinert werden. Das Bildungsministerium unterstützt die Qualifizierung mit einer Anrechnungsstunde und der Erstattung der Fahrtkosten für die Mentorinnen und Mentoren. Die Schulämter und Schulleitungen unterstützten durch Freistellungen und die Gestaltung der Stundenpläne für die Mentorinnen und Mentoren. Akteurinnen und Akteure aus den Fachwissenschaften, den Fachdidaktiken, den Bildungswissenschaften, dem Zentrum für Lehrerbildung und Bildungsforschung, der zweiten und dritten Phase beteiligten sich mit Weiterbildungselementen an der Qualifizierung. Weil Hochschule, Schule und Bildungsministerium als Systeme sehr unterschiedlich funktionieren, müssen für die Unterstützung der Mentorinnen und Mentoren geeignete Brückenstrukturen geschaffen werden. Beispielsweise ist in M-V der März ein guter Weiterbildungsmonat, weil die Prüfungszeit an den Schulen noch nicht angefangen hat und Praxisphasen in der Vorlesungszeit ab April stattfinden. Die Frage, wer welche Aufgabe am besten erfüllt und wie Strukturen dafür geschaffen werden können, begleitete unser Projekt während der gesamten Zeit.

\section{MQ nutzt für Entwicklung und stete Weiterentwicklung der Qualitäten ein mul- tiperspektivisches Team.}

In unserer Arbeit hat es sich als äußerst fruchtbar erwiesen, dass schon das Projektteam aus Fachdidaktikerinnen und -didaktikern verschiedener Fächer und Institutionen sowie der Schulpädagogik bestand. Schulischer Unterricht steht fast immer in Fachzusammenhängen, und Studierende werden bei ihrem Lernen in Fachpraktika begleitet - Fachmentoring (vgl. Staub \& Kreis 2013) ist demnach höchst hilfreich. Darüber hinaus sind die Praktika Lernorte „im dritten Raum“: Weitere beteiligte Akteurinnen und Akteure (Schulaufsicht, Schulleitung) werden idealerweise in die Qualitätsentwicklung eingebunden. 
5. MQ sichert ausreichende Ressourcen für die Tätigkeit der Mentorinnen und Mentoren ab (z.B.: Anrechnungsstunden, Fahrtkosten).

Mentoring ist eine wichtige Form der Lernbegleitung im Schulpraktikum. Aus diesem Grund darf es sich dabei keinesfalls um Schattenarbeit handeln, sondern sie muss angemessen remuneriert werden (Lehraufträge, Stundenabminderungen der Ausbildungsphase und auch danach), Auslagen sollten vergütet werden (Fahrt- und Materialkosten). Dazu gehören auch die Ressourcen für die eigene Weiterbildung der Mentorinnen und Mentoren.

\section{MQ wird mittelfristig in bestehende Strukturen eingebunden.}

Entwicklungsforschungsteams wie jenes der Mentor*innenqualifizierung $\mathrm{M}-\mathrm{V}$ können ihre Arbeit mit Ende einer Projektförderung nicht „on top“ ihres Tagesgeschäfts betreuen. Für die Kontinuität empfehlen wir die Anbindung an das Lehrkonzept bestehender Weiterbildungsstrukturen im jeweiligen Kontext (bspw. Institute für Qualitätsentwicklung usw.).

\subsubsection{Umsetzungsprinzipien}

Die Umsetzungsprinzipien (procedural principles) benennen laut Van den Akker (1999) konkrete Aktionsformen, die sich in der Entwicklungsstudie als wirksam im Hinblick auf das Erreichen der Lernziele herausgestellt haben. Die folgenden Umsetzungsprinzipien sagen also, wie Mentor*innenqualifizierungsprogramme auf der Basis unserer Erkenntnisse ganz konkret zu bauen sind:

7. MQ umfasst Strukturwissen, Selbstkompetenz, Betreuungskompetenz und gegenstandsbezogene Kompetenz.

Der Gegenstand einer Mentor*innenqualifizierung ist der Praxisphasentetraeder in allen Facetten. Daraus ergeben sich spezifische Weiterbildungsinhalte. Mentorinnen und Mentoren kennen und reflektieren ihre Rolle (Selbstkompetenz). Sie kennen die Strukturen, aus denen die Mentee in die Praxisphase kommen, damit an deren Entwicklung angeknüpft werden kann (Strukturwissen). Darauf aufbauend gestalten die Mentorinnen und Mentoren die Lernsituation durch passende Methoden wie Gesprächsführung und Coaching (Betreuungskompetenz). Für die Gestaltung der Praxisphasen gehen sie zudem auf die fachwissenschaftlichen, fachdidaktischen und allgemeinpädagogischen Gegenstände ein (gegenstandsbezogene Kompetenzen).

8. Allgemeine und gegenstandsbezogene Qualifizierungsanteile werden vernetzt und aufeinander bezogen (Inhalte, Arbeitsweisen).

In den Weiterbildungen zeigten sich insbesondere an den Stellen Erkenntnisse, an denen die Qualifizierungsschwerpunkte vernetzt wurden. Während ein Eingreifen 
der Mentorin oder des Mentors während des Unterrichtens von Studierenden beispielsweise grundsätzlich abgelehnt wurde, zeigte sich bei der Diskussion um das Experimentieren im Biologieunterricht die Notwendigkeit, an manchen Punkten einzugreifen (Nestler \& Retzlaff-Fürst i.V. a). Gesprächsübungen konnten durch die praktische Anwendung an Unterrichtsentwürfen aus dem eigenen Fach geübt werden. Die Vielfalt der Professionalisierungswege der Mentorinnen und Mentoren diente zur Auseinandersetzung mit der Professionalisierung der Mentees und der Entwicklung der eigenen Rolle.

9. MQ findet eine stimmige Balance zwischen Instruktion und moderiertem Austausch der Expertisen der Mentorinnen und Mentoren.

Mentorinnen und Mentoren sind Expertinnen und Experten des Lehrens und Lernens. Auch wenn sie Lernen bisher vor allem für Kinder und Jugendliche an Schulen modelliert haben sollten, sind sie keinesfalls als Beginnende anzusprechen. Das Qualifizierungsprogramm sollte stets nach einer guten Balance zwischen zu vermittelnden Inhalten und Formen des Austausches (z.B. über aktuelle Betreuungsarbeit) oder sogar Empowerment der Mentorinnen und Mentoren in professionellen Lerngruppen (Malmberg 2018c) suchen. Unsere Kursevaluationen zeigen an vielen Stellen, dass diese Balance regelmäßige Aufmerksamkeit erforderte und wirklich sensible Punkte bei den Mentorinnen und Mentoren traf. Hilfreich empfanden wir es dabei, das Gelingen der Balance punktuell zum Austauschthema mit den Mentorinnen und Mentoren zu machen. In der Evaluation des ersten Jahrganges verwies eine Mentorin oder ein Mentor auf diese Balance: "Ich habe sehr gerne an dieser Fortbildung teilgenommen, da ich nicht nur viel gelernt habe, sondern auch in Kontakt mit vielen Kolleg*innen gekommen bin und die Stimmung bei der Fortbildung als sehr angenehm, informativ und produktiv empfunden habe."

10. MQ bindet aktuelle und individuelle Professionalisierungsinteressen der Mentorinnen und Mentoren (hinsichtlich Mentoring und Unterricht) ein.

Die kompetente Betreuung angehender Lehrerinnen und Lehrer war ein zentraler Beweggrund, an der Mentor*innenqualifizierung teilzunehmen (17 Nennungen, $\mathrm{n}=35$, Evaluation Jahrgang 17/18). Unsere Evaluationen zeigen aber auch, dass einige Mentorinnen und Mentoren insbesondere durch ihr Interesse an der eigenen Professionalisierung unabhängig vom Mentoring teilnahmen (17 Nennungen, $\mathrm{n}=35$, Evaluation Jahrgang 17/18). Beispielsweise war es attraktiv, in das Lernbüro der Schulpädagogik für das Fach Deutsch oder die Diskussion über aktuelle Entwicklungen der Genetik in Biologie eingebunden zu sein, oder im Bereich Musik ganz einfach regelmäßigen Austausch mit engagierten Fachkolleginnen und -kollegen über Musik-Themen zu haben. Die Einbindung solcher Interessen 
in die Qualifizierung adressiert den Unterrichtstetraeder auf der untersten Ebene des Tetraedermodells (Abb. 5, hellgrau).

\section{MQ setzt Maßnahmen zur Anerkennung und zur Identitätsbildung der Mento- rinnen und Mentoren.}

Mentorinnen und Mentorinnen fördern durch ihre Rolle zwischen den Institutionen Hochschule und Schule den Wissenstransfer, sie selbst befinden sich in einer Doppelrolle als Vertreter beider Orte (Schullehrende und hochschulische Lehrende). Das gekonnte Einnehmen der anspruchsvollen Doppelrolle erfordert Maßnahmen zur Identitätsbildung (vgl. Kreis 2012; Malmberg \& Lion 2014, Malmberg 2018c). Die strukturellen Anerkennungen der Anrechnungsstunden und Freistellungen wurden ergänzt durch die Eröffnung des Qualifizierungsjahres durch die Direktorin des Zentrums für Lehrerbildung und Bildungsforschung sowie aus dem Ministerium für Bildung, Wissenschaft und Kultur. Der Abschluss der Qualifizierung erfolgte durch eine Zertifizierung. In der Bedeutung für die Mentorinnen und Mentoren war aber der gemeinsame Austausch und die Anerkennung durch eine Gesprächskultur der Augenhöhe mit den Lehrenden der Qualifizierung und Partnerinnen und Partnern der Hochschule mindestens ebenso wichtig. Die Etablierung professioneller Lerngruppen der Mentorinnen und Mentoren befördert die Identitätsbildung längerfristig.

12. MQ fördert das Verständnis über und die Unterstützung von individuellen Professionalisierungswegen der Mentees.

Mentorinnen und Mentoren benötigen Wissen und Verständnis dafür, dass auch ganz unterschiedliche Berufsanfängerinnen und -anfänger sich zu erfolgreichen Lehrenden entwickeln können. Professionalisierungswege verlaufen höchst individuell (Košinár 2018). Hilfreich werden ihnen dabei der Austausch in der professionellen Lerngruppe sowie Wissen und Materialien zu biografischen und selbstreflexiven Mentoringimpulsen. Zusätzlich helfen Klärungen, der eigenen Professionalisierungswege mit anschließenden Diskussionen oder Vergleichen. Insbesondere die Frage nach der Berufswahlsicherheit der Lehrerinnen und Lehrer (Beispielfrage: Zu welchem Zeitpunkt waren Sie sicher, dass Sie Lehrerin bzw. Lehrer werden wollten?) verdeutlichte die Individualität von Professionalisierungswegen.

13. Die verwendeten Medien sind qualitativ so ausgestattet, dass sie die Wirkungsketten unterstützen.

Über ausgegebene und/oder gemeinsam erstellte Materialien zum Mentoring oder zu weiteren Wissensinhalten können die Wirkungsketten (Abb.5, 3) sehr effektiv unterstützt werden. Zu den bekannten Merkmalen der Unterrichtsqualität (Meyer 2018, Helmke 2017) können dann auch fachspezifische Qualitätsmerkmale, 
Übersichten zur Klassenführung oder individuelle, fachspezifische Schwerpunkte hinzukommen. Wirkungsketten werden so zu Wirkungsnetzen. Idealerweise können gemeinsam erstellte Dokumente der Mentor*innenqualifizierung dann direkt in den Praxisphasen oder auch im Unterricht angewendet werden. Mentorinnen und Mentoren müssen dann nicht alle Theorien oder Qualitätsmerkmale wiedergeben, aber können sich bei Bedarf auf die übersichtlichen Materialien beziehen. So wird dem Vergessen einzelner Aspekte vorgebeugt.

\section{Die MQ nutzt geeignete Strategien, um langfristige professionelle Lerngruppen anzuregen (und nutzt dafür auch das inhärente Interesse an Kollegenaustausch).}

Der ursprüngliche Projektantrag fokussierte auf eine einmalige Ausbildung der Mentorinnen und Mentoren. Mentoring wurde dabei als eine zu erlernende Aufgabe von Lehrerinnen und Lehrern verstanden. Die Mentorinnen und Mentoren des ersten Qualifizierungsjahrganges fragten, ob sie auch im neuen Jahr teilnehmen können. Viele dieser Mentorinnen und Mentoren begleiteten die universitären Praxisphasen dabei schon für einige Jahre. Wir beobachteten, dass die Heterogenität der Mentorinnen und Mentoren in den Diskussionen immer wieder dafür sorgte, dass neue Themen auf die Tagesordnung kamen. Die Gestaltung von Unterrichtsentwürfen, die Frage nach der Qualität von Unterricht und die Bildung der eigenen Rolle zeigten vielfältige Wege der Betreuung. Diese Diskussionen wurden zum essenziellen Bestandteil der Weiterentwicklung der Mentor*innenqualifizierung.

Für die neuen Mentorinnen und Mentoren in den professionellen Lerngruppen wirkte es bereichernd, so verschiedene Kolleginnen und Kollegen zu sehen. Gleichzeitig verschmolz die Grenze zwischen Qualifizierenden, also Fachdidaktikerinnen und Fachdidaktikern, und Mentorinnen und Mentoren. Das Ziel der bestmöglichen Lernbegleitung für Praxisphase und Unterricht rückte damit stärker in den Fokus der professionellen Lerngruppen. Durch das gemeinsame Gestalten von Materialien, begleitende Gesprächsübungen und Rollenreflexionen bleibt die Vernetzung (Designprinzip 8) in den Weiterbildungen aufrechterhalten. Die Tiefe der Diskussionen wurde durch Mentorinnen und Mentoren gesteigert, die länger im Qualifizierungsprogramm waren. Aus diesem Grund wurde beschlossen, dass der Aufbau professioneller Lerngruppen angestrebt wird. 


\section{Gegenstandsbezogene Lernbegleitung in Biologie}

Am Beispiel der fachspezifischen Qualifizierung für Biologiementorinnen und -mentoren werden nun einige der Gestaltungsprinzipien und deren Umsetzung exemplarisch erläutert. Zuerst betrachten wir eine konkrete Unterrichtssituation. Wir befinden uns damit in der untersten Ebene des Tetraedermodells (Abb. 2 bis 5, hellgrau). Die Schülerinnen und Schüler sollen im Biologieunterricht mit Hilfe von naturwissenschaftlich-biologischen Arbeitsweisen Erkenntnisse gewinnen (Vgl. KMK 2004; Mayer 2018). Beispielweise sollen sie mikroskopieren, experimentieren oder Lebewesen bestimmen. Die Entwicklung im Kompetenzbereich Erkenntnisgewinnung wird in verschiedenen Themengebieten gefördert. Beispielsweise ist das Mikroskopieren und Zeichnen einer Zwiebelzelle im Themenfeld Zytologie ein häufiges Unterrichtsziel (vgl. Abb. 5, A).

Auf der mittleren Ebene des Tetraedermodells wird die Lehrerbildung in den Blick genommen (Abb. 2 bis 5, dunkelgrau). Studierende müssen in Bezug auf die beschriebene Unterrichtssituation (mindestens) zwei Kompetenzen erwerben (vgl. Abb. 5, C).

Die Studierenden sollen

I. aus den im Rahmenplan geforderten Kompetenzen konkrete Unterrichtsziele ableiten.

II. eine Unterrichtsstunde erstellen können, die das Gewinnen von Erkenntnissen mit Hilfe der Mikroskopie ermöglicht.

Die Entwicklung der genannten Kompetenzen der Studierenden wird zum Gegenstand des Lehramtsstudiums. In der Vorlesung „Fachdidaktik Biologie“ und dem zugehörigen Begleitseminar wird das Wissen zum Erlangen dieser Kompetenzen erworben und angewandt. In der Praxisphase Schulpraktische Übungen sollen die Studierenden das erworbene Wissen anwenden und in der praktischen Umsetzung die zugehörigen Fähigkeiten und Fertigkeiten ausbilden. Die Mentorinnen und Mentoren unterstützen die Studierenden dabei.

Für die Gestaltung der Qualifizierung von Mentorinnen und Mentoren (Abb. 2 bis 5, schwarz) können nun die Leit- und Umsetzungsprinzipien genutzt werden. Das Meta-Designprinzip $1-M Q$ adressiert die ebenenübergreifenden Wirkungsketten. - fordert einerseits den Fokus auf einzelne Wirkungen und andererseits den Blick auf die Vernetzung dieser Wirkungen.

Im ersten Jahrgang 2017/18 war das Ableiten von konkreten Unterrichtszielen aus den Kompetenzen (Ziel I.) ein Gegenstand der Mentor*innenqualifizierung Biologie. Dieser Qualifizierungsteil startete mit der Einführung zu „Zielen und Kompetenzen im Biologieunterricht". Anschließend diskutierten und erweiterten die Mentorinnen und Mentoren eine neu konzipierte Übersicht zu diesem 
Schwerpunkt, sodass in Vorlesung und Praxisphase mit dem gleichen Material gearbeitet werden kann. Dadurch wurde die stimmige Balance zwischen Instruktion und moderiertem Austausch des Expertenwissens (Designprinzip 9) gesucht und Medien so gestaltet, dass sie die Wirkungsketten unterstützen (Designprinzip 14). Die Mentorinnen und Mentoren wendeten das Gelernte und die Materialien in anschließenden Übungen zur ko-konstruktiven Gesprächsführung an. Gegenstandsbezogene Kompetenzen und Betreunngskompetenz (Designprinzip 7) wurden so vernetzt entwickelt (Designprinzip 8).

Im Jahrgang 2018/19 war das Ziel II. Erkenntnisgewinnung durch das Mikroskopieren Gegenstand der Mentor*innenqualifizierung Biologie. Die Möglichkeiten beim Mikroskopieren sind für eine wissenschaftliche Einrichtung deutlich höher als bei der schulischen Mikroskopie, die durch strukturelle (Es sind nur Lichtmikroskope verfügbar.) und gesellschaftliche (Der Rahmenplan fordert insbesondere Lichtmikroskopie.) Einschränkungen geprägt wird. Somit ist Mikroskopieren ein Bestandteil der Fachlichkeit, bei dem wissenschaftliches und schulisches Bild verschieden sind. Damit die Mentorinnen und Mentoren in dieser Fachlichkeitskonstruktion vermitteln können (Designprinzip 2), wurden die Fachakteurinnen und -akteure aus dem Mikroskopiezentrum in der Stadt mit einbezogen (Designprinzip 3). Am Vormittag eines Qualifizierungstages setzten sich die Mentorinnen und Mentoren mit ihren zukünftigen Mentees mit den Grenzen und Möglichkeiten der Mikroskopie als naturwissenschaftlich-biologische Arbeitsweise auseinander. Dadurch adressierte die Qualifizierung zum einen auch individuelle Professionalisierungsinteressen der Mentorinnen und Mentoren (Designprinzip 10). Zum anderen hatten sich die Mentorinnen und Mentoren gewünscht (Designprinzip 3), die Mentee schon vertieft vor der Praxisphase kennenzulernen. Nach dem gemeinsamen Mikroskopieren folgte die Reflexion zu der Frage: Wie können die gemeinsamen Erkenntnisse in der Praxisphase und im dazugehörigen Unterricht angewandt werden? In diesem Fall arbeiteten Mentorinnen und Mentoren, Mentee, Fachdidaktikerinnen und Fachdidaktiker und Fachwissenschaftlerinnen und Fachwissenschaftler (Designprinzip 3) an den ebenenübergreifenden Wirkungsketten (Designprinzip 1).

In der Zusammenschau dieser beiden Weiterbildungsabschnitte aus dem Jahrgang 2017/18 und 2018/19 für das Fach Biologie sieht man das Zusammenspiel verschiedener Designprinzipien. Durch den Fokus auf ganz spezifische Wirkungen und deren Zusammenhänge in Wirkungsketten können eine Kohärenz in der Lehrerbildung und die Entwicklung der ebenenübergreifenden Lernbegleitung leichter angestrebt werden. 


\section{Ausblick}

Die vorgestellte Studie leistet einen Beitrag zur Frage, wie Lehrerbildung in schulischen Praxisphasen weiterentwickelt werden kann. Eine Wirksamkeit der Mentor*innenqualifizierung auf allen Ebenen des Lernens (Mentoring, Studierende, Schülerinnen und Schüler) wird dezidiert angestrebt (Designprinzip 1 Wirkungsketten), sie ist jedoch empirisch noch nicht abgesichert. Mit Blick auf die Forderung nach Veränderungen der Strukturen (Designprinzip 6) und der Bereitstellung ausreichender Ressourcen (Designprinzip 5) sollten in einem nächsten Schritt Teilaspekte der Wirkungsketten in näherer Zukunft empirisch überprüft werden.

Für die Mentor*innenqualifizierung im Fach Biologie stellen sich beispielsweise Fragen danach, ob sich durch die Mentor/Mentee-Besprechungen auch schulische Unterrichtsziele verändern. Es bleibt außerdem weiterhin offen, ob eine Mentor*innenqualifizierung tatsächlich die Entwicklung der Kompetenzen der Studierenden fördert (Nestler \& Retzlaff-Fürst in Vorb.). Und es stellt sich die Frage nach dem Zusammenhang zwischen der Mentor*innenqualifizierung und dem Lernen von Schülerinnen und Schülern in Praktikumsschulen.

Das Modell des gegenstandsbezogenen Mentoring erfüllt somit zwei Funktionen. Erstens klärt es bei der Konzeptionierung einer Mentor*innenqualifizierung den Blick auf die einzelnen Wirkungsketten. Zweitens eröffnet es konkrete Fragen für die empirische Bildungsforschung, wie Wirkungsketten tatsächlich funktionieren.

Vor nun möglicherweise anschließenden Studien stellt sich jedoch noch die wichtige Frage, welche Kompetenzen Studierende in Praxisphasen eigentlich entwickeln sollen (eine Frage, die für die Ebene des Schülerinnen- und Schülerlernens besser beantwortet ist, für Studierende jedoch nur äußerst heterogen oder informell). Vor dem Hintergrund der vielen Fächer, Fachdisziplinen sowie gesellschaftlichen Herausforderungen wie Digitalisierung und Umgang mit Heterogenität droht aus unserer Sicht eine Überfrachtung der Praxisphasen. Nach einer Klärung der angestrebten Ziele von Praxisphasen für die Studierenden kann eine Mentor*innenqualifizierung mit den oben genannten Designprinzipien gezielter gestaltet werden. 


\section{Isolde Malmberg, Emanuel Nestler und Carolin Retzlaff-Füirst}

\section{Literatur}

Bakker, Arthur (Hrsg.) (2019): Design research in education. A practical guide for early career researchers. Abingdon, Oxon, New York: Routledge.

Bakker, Arthur (2019): Chapter 9. Design Principles in design research: A commentary. In: Bikner-Ahsbahs, A./Maria, P. (Hrsg.): Unterrichtsentwicklung macht Schule. Fachdidaktische Forschung und Innovation im Fachunterricht Wiesbaden: Springer, 177-192.

Baumert, Jürgen \& Kunter, Mareike (2006): Stichwort: Professionelle Kompetenz von Lehrkräften. In: Zeitschrift für Erziehungswissenschaft, 9. Jg., Nr. 4, 469-520.

Cobb, Paul; Confrey, Jere; diSessa, Andrea; Lehrer, Richard \& Schauble, Leona (2003): Design Experiments in Educational Research. In: Educational Researcher. 32. Jg., Nr. 1, 9-13.

Euler, Dieter (2014a): Design Principles als Kristallisationspunkt für Praxisgestaltung und wissenschaftliche Erkenntnisgewinnung. In Euler, D./Sloane, P. (Hrsg.): Design-Based Research. Stuttgart: Franz Steiner, 97-112.

Euler, Dieter (2014b): Design-Research - a paradigm under development. In: Euler, D./Sloane, P.F.E. (Hrsg.): Design-Based Research Stuttgart: Franz Steiner, 15-44.

Euler, Dieter (2017): Design-Principles as bridge between scientific knowledge production and practice design. In: Educational Design Research, Vol. 1, Issue 1, Article 02.

Euler, Dieter \& Sloane, Peter F. E. (2018): Design-Based Research. In: Rauner, F./Grollmann, P. (Hrsg.): Handbuch Berufsbildungsforschung. Bielefeld: wbv, 782-790.

Gröschner, Alexander; Müller, Katharina; Bauer, Johannes; Seidel, Tina; Prenzel, Manfred; Kauper, Tabea \& Möller, Jens (2015): Praxisphasen in der Lehrerausbildung - Eine Strukturanalyse am Beispiel des gymnasialen Lehramtsstudiums in Deutschland. In: Zeitschrift für Erziehungswissenschaft, 18. Jg., Nr. 4, 639-665.

Gröschner, Alexander \& Schmitt, Cordula (2009): Skalen zur Erfassung von Kompetenzen in der Lehrerausbildung. Ein empirisches Instrument in Anlehnung an die KMK „Standards für die Lehrerbildung: Bildungswissenschaften“. Jena.

Helmke, Andreas (2017): Unterrichtsqualität und Lehrerprofessionalität. Diagnose, Evaluation und Verbesserung des Unterrichts. Seelze: Klett-Kallmeyer.

Helmke, Andreas; Tuyet, Lenske; Gerlinde; Pham, Giang, Praetorius, Anna-Katharina; Schrader, Friedrich-Wilhelm \& Ade-Thurow, Manuel (2018): Unterrichtsdiagnostik mit EMU. Evidenzbasierte Methoden der Unterrichtsdiagnostik und -entwicklung. Version 7.0. Universität Koblenz-Landau. Online unter: http://unterrichtsdiagnostik.de/media/files/Broschuere_Version_7.0.pdf. (Abrufdatum: 20.07.2019).

KMK, Kultusministerkonferenz (2004): Bildungsstandards im Fach Biologie für den Mittleren Schulabschluss. Online unter: https://www.kmk.org (Abrufdatum: 13.03.2018)

Košinár, Julia (2018): das Mentorat zwischen Individualisierung und Standardisierung - eine empirie und theoriebasierte Konzeption. In: Reintjes, C./Bellenberg, G./im Brahm, G. (Hrsg.): Mentoring und Coaching als Beitrag zur Professionalisierung angehender Lehrpersonen (=Schulpraktische Studien und Professionalisierung Bd.3). Münster, New York: Waxmann, 67-84.

Kreis, Annelies (2012): Produktive Unterrichtsbesprechungen. Lernen im Dialog zwischen Mentoren und angehenden Lehrpersonen. Schulpädagogik-Fachdidaktik-Lehrerbildung, Band 17. Bern, Stuttgart, Wien: Haupt Verlag.

Malmberg, Isolde \& Lion, Brigitte (2014). Begleitung an der Schnittstelle. Die MentorInnen im Lehramtsstudium der mdw: Aufgaben, Rollen und Selbstverständnis. In: Schrittesser, I./Malmberg, I./ Mateus-Berr, R./Steger, M. (Hrsg.): Zauberformel Praxis? Zu den Möglichkeiten und Grenzen von Praxiserfahrungen in der LehrerInnenbildung. Wien: new academic press, 205-218.

Malmberg, Isolde (2018a). Transitions between Art and Pedagogy. Mentoring Music Teacher Novices in Austria. In: Global Education Review XX Vol 4 No 4 (2017), 39-53. New York: Mercy College. Unter: http://ger.mercy.edu/index.php/ger/article/view/294 (Abrufdatum: 27.11.2019). 
Malmberg, Isolde (2018b): Auf einer mehrspurigen Autobahnbrücke. Die Statuspassage am Beginn des Musiklehrberufs. In: Schäfer-Lembeck, U./Zöllner-Dressler, S./Krettenauer, T. (Hrsg.): BFG-kontakt, 297-309.

Malmberg, Isolde (2018c): Musikpädagogischen Eigensinn entwickeln helfen. Zum Empowermentprozess der MentorInnen an der Wiener Musikuniversität und Auswirkungen auf deren Betreuungsarbeit. In: Aigner, W./Christof, E. (Hrsg.): schulheft 4/2018. 54-67.

Malmberg, Isolde; Nestler, Emanuel \& Piotraschke, Maximilian (2018): Mentor*innenqualifizierung aus fachdidaktischer Perspektive gestalten. Kooperative Entwicklung und Umsetzung eines Curriculums. In: Journal für LehrerInnenbildung 4/2018. 46-51.

Malmberg, Isolde (i.V.): Entwicklungsforschung in Musik. Überlegungen zu fachbezogenen Aspekten eines Forschungsstils. In: Lehmann-Wermser, A./Konrad, U. (Hrsg.): Musikpädagogische Forschung zwischen Theoriebildung und Praxisveränderung. Hannover.

Martens, Matthias; Rabenstein, Kerstin; Bräu, Karin; Fetzer, Marei; Gresch, Helge; Hardy, Ilonca \& Schelle, Carla (Hrsg.) (2018): Konstruktionen von Fachlichkeit. Ansätze, Erträge und Diskussionen in der empirischen Unterrichtsforschung. Bad Heilbrunn: Julius Klinkhardt.

Mayer, J. (2018): Erkenntnisse mit naturwissenschaftlichen Methoden gewinnen. In: Gropengießer, H./Harms, U./Kattmann, U. (Hrsg.): Fachdidaktik Biologie. Hallbergmoos, Aulis, 114-124.

McKenney, Susan \& Reeves, Thomas C. (2012): Conducting Educational Design Research. New York: Routledge.

Meyer, Hilbert (2018): Was ist guter Unterricht? Berlin: Cornelsen Verlag Scriptor.

Nestler, Emanuel \& Retzlaff-Fürst, Carolin (i.V. a): Die Mentor*innenqualifizierung Biologie zur Unterstützung der Reflexion von fachwissenschaftlichen und fachdidaktischen Konzepten Studierender

Nestler, Emanuel \& Retzlaff-Fürst, Carolin (i.V. b): Naturwissenschaftlich-Biologische Erkenntnisgewinnung in Praxisphasen: eine Interventionsstudie zur Qualifizierung von Mentorinnen und Mentoren für die Betreuung von Biologielehramtsstudierenden.

Niggli, Alois (2005): Unterrichtsbesprechungen im Mentoring. Oberentfelden: Sauerländer.

Prediger, Susanne; Link, Michael; Hinz, Renate; Hußmann, Stephan; Thiele, Jörg \& Ralle, Bernd (2012): Lehr-Lernprozesse initiieren und erforschen - Fachdidaktische Entwicklungsforschung im Dortmunder Modell. In: Der mathematische und naturwissenschaftliche Unterricht, 65 (2012), 452-457. Online unter http://www.mathematik.uni-dortmund.de/-prediger/veroeff/12-Prediger_et_al_MNU_FUNKEN_Webversion.pdf (Abrufdatum: 20.3.2019).

Prediger, Susanne; Link, Michael; Hinz, Renate; Hußmann, Stephan; Thiele, Jörg \& Ralle, Bernd (2012): Lehr-Lernprozesse initiieren und erforschen - Fachdidaktische Entwicklungsforschung im Dortmunder Modell. In: MNU 65(8), 452-457

Prediger, Susanne; Leuders, Timo \& Rösken-Winter, Bettina (2017): Drei-Tetraeder-Modell der gegenstandsbezogenen Professionalisierungsforschung: Fachspezifische Verknüpfung von Design und Forschung. In: Jahrbuch für Allgemeine Didaktik, 159-177.

Reinmann, Gabi (2005): Innovation ohne Forschung? Ein Plädoyer für den Design-Based Research-Ansatz in der Lehr-Lernforschung. In: Unterrichtswissenschaft 33(1), 52-69.

Reinmann, Gabi (2018): Reader zu Design-Based Research. Hamburg. Online unter https://gabi-reinmann.de/wp-content/uploads/2018/06/Reader_DBR_Juni2018.pdf (Abrufdatum: 1.7.2019).

Schnebel, Stefanie (2018): Welche Qualifizierung brauchen Praxislehrpersonen? Überlegungen, Kenntnisse und Kompetenzen. In: Journal für Lehrerinnenbildung, 18. Jg., Nr. 4, 22-27.

Schüpbach, Jörg (2005). Die Unterrichtsnachbesprechung in den Lehrpraktika - eine „Nahtstelle von Wissen und Handeln“?. Eine deskriptiv-empirische Studie zur Bedeutung der Reflexion im Theorie-Praxis-Bezug in der Lehrerbildung. Zürich: Universität Zürich.

Staub, Fritz C. \& Kreis, Annelies (2013): Fachspezifisches Unterrichtscoaching in der Aus- und Weiterbildung von Lehrpersonen. In: Journal für Lehrerinnenbildung, 2. Jg., Nr. 13, 8-13. 


\section{Isolde Malmberg, Emanuel Nestler und Carolin Retzlaff-Füirst}

Van den Akker, Jan (1999): Principles and methods of development research. In: Van den Akker, J./Nieveen, N./Branch, R.M./Gustafson, K./Plomp, T. (Hrsg.): Design approaches and tools in education and training. Dordrecht: Springer, 1-14.

Van den Akker, J.; Gravemeijer, K.; McKenney, S. \& Nieveen, N. (Hrsg.) (2006): Educational Design Research. London: Routledge.

Zeichner, Ken (2010): Rethinking the Connections Between Campus Courses and Field Expieriences in College- and University-Based Teacher Education. In: Journal of Teacher Education, 61. Jg., Nr. 1-2, S. 89-99. 